\title{
Elderly-Onset Multiple System Atrophy with Lewy Body Pathology: A Case Report
}

\author{
Kazuhiro Fukushima ${ }^{a} \quad$ Akinori Nakamura ${ }^{a}$ Yo-ichi Takei ${ }^{a}$ Kenya Oguchia \\ Hiroko Itagaki $^{b}$ Shinji Ohara ${ }^{a, c}$ Mitsunori Yamada ${ }^{d}$ \\ aDepartment of Neurology, National Hospital Organization, Matsumoto Medical Center, \\ Matsumoto, Japan; bepartment of Diagnostic Pathology, National Hospital Organization, \\ Matsumoto Medical Center, Matsumoto, Japan; 'Department of Neurology, lida Hospital, \\ lida, Japan; dDepartment of Brain Disease Research, Shinshu University School of Medicine, \\ Matsumoto, Japan
}

\section{Keywords}

Multiple system atrophy $\cdot$ Parkinson's disease $\cdot \alpha$-synucleinopathy $\cdot$ Neurodegeneration

\section{Abstract}

An 81-year-old woman presented with a 2-year history of progressive dysarthria and gait disturbance. Subsequently, she developed orthostatic hypotension, obstructive sleep apnea, right-sided resting tremor, and rigidity. Together with characteristic findings of imaging studies, she was diagnosed with multiple system atrophy (MSA). Despite progressive dysphagia and repeated choking episodes, the patient elected not to use artificial feeding or tracheostomy. She died suddenly at age 91 after 12 years of illness. The autopsy revealed neuropathological features of both MSA and of Parkinson's disease. The peripheral autonomic ganglia revealed both pre- and postganglionic involvement by synucleinopathy, which may have underscored the sudden death of the patient. The patient survived 10 years after onset, despite the presence of multiple poor prognostic factors in MSA including the onset of old age and early appearance of orthostatic hypotension and falls, in addition to the complication of PD pathology found by autopsy. Multidisciplinary team approach and her preserved cognitive function may have been contributory to the long-term survival. 


\section{Introduction}

Multiple system atrophy (MSA) is an adult onset, fetal neurodegenerative disease characterized by various combinations of parkinsonism, ataxia, and autonomic failure [1, 2]. Previous studies revealed that the average disease onset is relatively constant in mid-fifties (54.2 56.2-years old), while mean duration of the illness varies among studies ranging from 5.5 to 9.8 years [2-6]. An average survival of 9 years in 230 patients has been reported from Japan [4].

Clinically, overlapping features with PD often cause diagnostic difficulties, especially in the early stages when the patients presents with parkinsonian features such as resting tremor. Pathologically, both MSA and PD belong to synucleinopathy but with different cytopathological features. MSA is characterized by the appearance of glial cytoplasmic inclusions (GCIs) in oligodendrocytes, while PD by those of Lewy bodies and Lewy neurites [7]. Although considered as 2 distinctive forms of synucleinopathy, Lewy body pathology is known to often coexist in MSA $[8,9]$. Here, we report an unusual case of clinically diagnosed as MSA with very advanced age of onset and the duration of illness of 12 years and proved to have both MSA and PD by postmortem examination.

\section{Case Report}

An 81-year-old Japanese woman presented with a 2-year history of progressive gait unsteadiness with occasional falls. She had been treated for hypertension and coronary heart disease and had a history of bilateral high tibial osteotomy for knee osteoarthritis. There was no family history of neurological disease.

On examination, her blood pressure was $117 / 80 \mathrm{~mm} \mathrm{Hg}$ and there was no orthostatic change. However, she reported that she often complained of vagueness of her consciousness, especially toward the evening, when her systolic blood pressure was found dropped to about $80 \mathrm{~mm}$ Hg. Her mental status was normal. Her speech was dysarthric. The extraocular movements were in full range with saccadic smooth pursuit without nystagmus. Muscle strength and muscle tone were normal throughout. Finger-to-nose testing revealed mild dysmetria. Sensory examination was normal. Her gait was mildly broad based with small steps. Deep tendon reflexes were normal, and there were no pathological reflexes.

Two years later from the first visit, she developed night stridor. She was diagnosed with obstructive sleep apnea and prescribed a nocturnal NPPV device. At that time, her neurologic examination revealed orthostatic hypotension, marked dysarthria, resting and action tremor of the right hand, and cogwheel rigidity of the neck and the distal upper extremities on the right side. She also had a freezing of gait. Magnetic resonance imaging showed an atrophy of the cerebellum and the brain stem, a "hot cross bun" sign in the pons, and linear high density in the lateral part of the putamen on T2-weighted sequences (Fig. 1). Dopamine transporter scan revealed decreased signal in the basal ganglia on the both sides (Fig. 1). Her symptoms did not respond to the administration of trial dose L-dopa (up to $300 \mathrm{mg} /$ day); however, the subsequent administration of pramipexole $(0.375 \mathrm{mg} /$ day $)$ resulted in a slight improvement in the tremor. Although orthostatic hypotension was treated with L-threo-DOPS, its dose had to be limited due to supine hypertension.

In the following year, she fractured her left hip after a fall and underwent total hip replacement surgery. She became wheelchair bound, and she started to use indwelling urinary catheter.

At age 87 years, she started having myoclonus in the extremities, which was favorably treated with clonazepam. She developed progressive anarthria and had frequent choking 

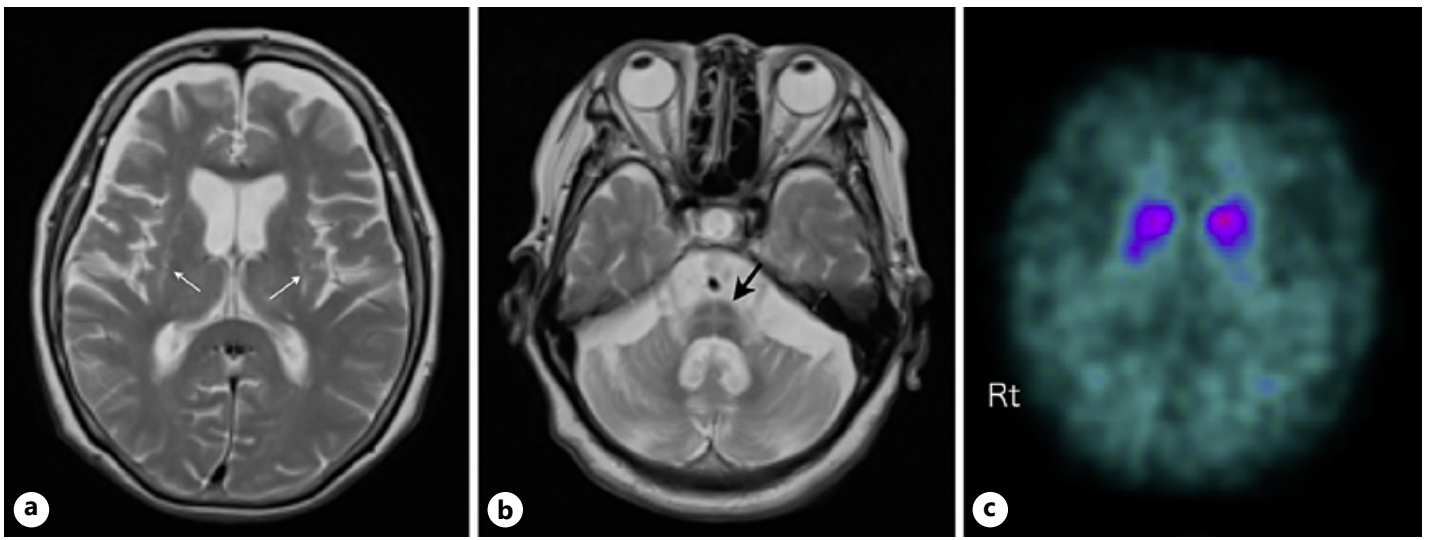

Fig. 1. Radiologic findings. a, b Brain T2-weighted MRI images, revealing a slit-like hyperintense rim bordering the putamen on both sides (arrows in a) and a "hot cross bun" sign in the pons (arrow in b). c DAT SPECT (DaT scan) showed severely decreased uptake in the basal ganglia on both sides. MRI, magnetic resonance imaging; DAT, dopamine transporter.

episodes on food. Her mental status was still normal, and she was able to communicate using a Hiragana letter board. She opted not to use tube feeding and declined tracheostomy.

At age 90 years, she was found having cardiorespiratory arrest early morning at home while using NPPV. Her family members had last seen her in her usual state only $5 \mathrm{~h}$ before. She was brought in our hospital and was pronounced dead. An autopsy was granted. General autopsy revealed organized of pneumonias and mild atherosclerotic changes in the visceral organs. No evidence of mechanical airway obstruction by sputum or food etc. could be found.

\section{Neuropathological Findings}

The brain was small and weighed $900 \mathrm{~g}$ post-fixation. Macroscopically, the cerebellum and pons were atrophic. The coronal section revealed that the bilateral putamen and globus pallidus were atrophic bilaterally, showing brownish discoloration most accentuated at the posterolateral part of the putamen. In the brain stem, the substantia nigra and the locus ceruleus were depigmented. The pontine base and middle cerebellar peduncle were markedly atrophic.

Formalin-fixed, paraffin-embedded sections of the brain and the spinal cord were stained with hematoxylin and eosin and Klüver-Barrera. Immunohistochemistry with a streptavidinbiotin method was performed with the monoclonal antibody against phosphorylated $\alpha$-synuclein antibodies (1:5,000; WAKO, Osaka, Japan; 1:5,000 LB509 Santa Cruz Biotech, Santa Cruz, Paso Robles, CA, USA). Sections of midbrain from a case of idiopathic Parkinson's disease harboring numerous Lewy bodies were used as positive control. Reaction products were visualized with DAB.

Histologically, severe neuronal loss and gliosis were found in the lateral part of the putamen, substantia nigra, locus ceruleus, pontine nuclei, inferior olivary nuclei, and Purkinje cells. With synuclein immunohistochemistry, $\alpha$-synuclein immunoreactive GCIs, and neuronal cytoplasmic inclusions were evident in these degenerated areas, except for Purkinje cells (Fig. 2a-d), confirming the diagnosis of MSA. In addition, Lewy bodies were present in the remaining neurons of the substantia nigra and locus ceruleus (Fig. 2d). The dorsal vagal nuclei in the medulla oblongata, a predilection site for Parkinson's disease, revealed mild neuronal loss with a few Lewy bodies and a few synuclein positive neurites (Lewy neurites). The 
cerebral cortices were well-preserved without Lewy bodies. The dentate nucleus and superior cerebellar peduncles were well-preserved.

In the sympathetic autonomic ganglia, there were many swollen neuronal processes and occasional Lewy bodies, which were immunoreactive for phosphorylated $\alpha$-synuclein (Fig. 2e-h). These $\alpha$-synuclein positive structures were occasionally found within the cytoplasm of ganglion cells (Fig. 2g), in the presynaptic terminals surrounding the neurons (Fig. 2h). The final neuropathological diagnosis was advanced MSA and PD (Braak stage III [10]).

\section{Discussion}

Although both MSA and PD are classified as synucleinopathies, they are separate disease entities. Interestingly however, coexistence of both diseases has been known to occur. Ozawa et al. [8] reported that brain stem Lewy body pathology was found in 10\% of 100 MSA cases, while Jellinger reported brain stem Lewy body pathology in $22.7 \%$ of 44 postmortem-proven MAS cases, compared to $8.4 \%$ of age-matched controls [9]. MSA and PD have overlapping clinical features, and it is difficult to clinically distinguish between them, and only autopsy can prove their coexistence $[2,11]$. As far as we are aware, this is the oldest case that was pathologically proven to have both MSA and PD. Based on the pathological findings, it was presumed that MSA pathology developed first, followed by PD pathology in this case. Similar to our case, Mochizuki et al. [12] reported an 82-year-old patient diagnosed with PD (onset case at age 79) whose autopsy findings unexpectedly revealed consistent with PD and an early stage of MSA.

Sudden death is common in the patients with MSA, which usually occurs during sleep, and is therefore attributed to suffocation resulting from vocal cord abductor paralysis, a characteristic laryngeal finding of this disease. That led to the use of tracheostomy and NPPV for the prevention of sudden death [13]. However, these treatments do not always prevent sudden death in MSA patients [14]. Sudden death in MSA is recently hypothesized to be a consequence of disordered central respiration, suffocation caused by sputum and food, upper airway obstruction from NPPV acting on a floppy epiglottis, cardiac autonomic disturbance, or a combination of these factors [13]. In the present case, the direct cause of sudden death could not be determined by autopsy. Based on the anatomical findings of both pre- and post-

Fig. 2. Neuropathological findings. a-d Upper pons, and $\mathbf{e}-\mathbf{h}$ paravertebral sympathetic ganglion. a Transverse section at the level of the upper pons, showing severe degeneration of transverse fibers in the basis pontis. Of note, the superior cerebellar peduncles $(*)$ are well-preserved in volume. KB myelin staining. Bar $=2 \mathrm{~mm}$. b $\alpha$-synuclein immunohistochemistry showing appearance of numerous GCIs in the pyramidal pathways in the area indicated by arrow in a. Bar $=20 \mu \mathrm{m}$. c This photo shows the rectangular area in a stained by $\alpha$-synuclein immunohistochemistry. Loss of neurons of the locus ceruleus is evident. (*): fourth ventricle. SCP: superior cerebellar peduncle. Bar $=200 \mu \mathrm{m}$. d Enlarged photo of the area in the rectangle of (c) showing Lewy bodies (long arrows) and a probable NCI (short arrow). Several GCIs are also seen (arrowheads). Bar $=50 \mu \mathrm{m}$. e, $\mathbf{h}$ Serial sections of the paravertebral sympathetic ganglia stained with H\&E. e and $\alpha$-synuclein immunohistochemistry (f), showing numerous swollen, Lewy body-like eosinophilic cell processes, which are synuclein immunoreactive (arrows). One of them (arrowhead) only shows weak marginal immunoreactivity. Bar $=20 \mu \mathrm{m}$. g $2 \alpha$-synuclein positive intracytoplasmic inclusions with a spiky appearance are seen in a sympathetic ganglion neuron. $\mathbf{h}$ This photo illustrates a ganglion neuron surrounded by swollen, $\alpha$-synuclein positive presynaptic terminals. e Bar $=20 \mu \mathrm{m}$. f-h Bar $=20 \mu \mathrm{m}$. GCI, glial cytoplasmic inclusion; NCI, neuronal cytoplasmic inclusion; H\&E, hematoxylin and eosin; KB, Klüver-Barrera.

(For figure see next page.) 
ganglionic autonomic involvement by synucleinopathy, it is speculated that peripheral and/ or central autonomic disturbance may have been responsible for sudden death in addition to upper airway obstruction associated with NPPV [14].

There are several key features in predicting the poor prognosis of the MSA patients. Watanabe et al. [4] studied 230 cases and reported that subgroup of MSA with shorter times from onset to concomitant motor and autonomic system impairment, particularly $<3$ years,

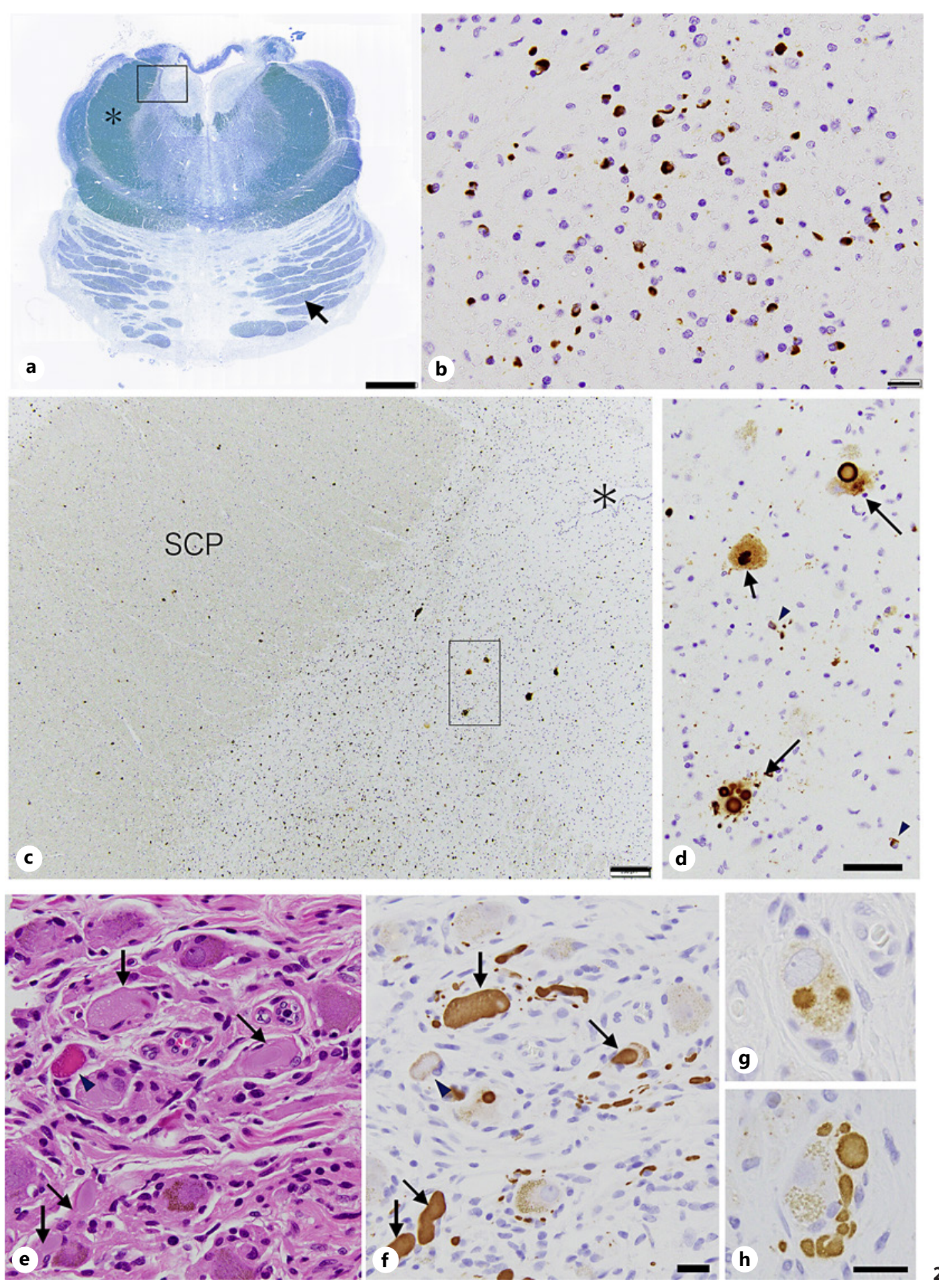


and onset after 55-year-old progressive more rapidly[6]. Coon et al. [6] analyzed large MSA series (685 cases) and identified 6 factors predicting poor prognosis: (i) falls within 3 years of onset; (ii) bladder symptoms; (iii) urinary catheterization within 3 years of symptom onset; (iv) orthostatic intolerance within 1 year of symptom onset; (v) older age of onset; and (vi) degree of autonomic failure as measured by a validated composite autonomic severity score. It may be noteworthy that the present patient survived 12 years after onset, despite the presence of several poor prognostic factors as described above, including the elderly onset of the illness and early onset of orthostatic hypotension and falls, in addition to the complication of PD pathology as found by autopsy.

What are the factors that have been responsible for our patient's comparatively longer survival? Shimohara et al. [13] emphasized importance of the multidisciplinary team approach to provide the best means to prevent sudden death in MSA. In this patient, in addition to the devoted care offered by her family members, the primary care physician, home-visiting nurses, dentists specializing swallowing evaluation, visiting physical and occupational therapists, and hospital neurologists worked cooperatively in taking care of the patient at home. In addition, we feel it significant that the patient's cognitive function was well-persevered until the end of her life, which enabled her to communicate with family members and medical staff.

\section{Acknowledgements}

The authors thank K. Asakawa, Department of Clinical Laboratory, Matsumoto Medical Center, for his technical assistance. We also thank Prof. R.E. Schmidt, Washington University School of Medicine in St. Louis, for critical reading of the manuscript.

\section{Statement of Ethics}

Written informed consent for publication was obtained from the patient's daughter (next of kin) for publication of this case report and accompanying images.

\section{Conflict of Interest Statement}

The authors report no conflicts of interests.

\section{Funding Sources}

The authors declare they did not receive any funding.

\section{Author Contributions}

K. Fukushima and S. Ohara were responsible for collection and interpretation of data, neuropathological examination, and drafting and revising the manuscript. A. Nakamura, $\mathrm{K}$. Oguchi, and Y. Takei were responsible for collection and interpretation of data, and revising the intellectual content of the manuscript. H. Itagaki and M. Yamada were responsible for neuropathological examination and revising the portion of the manuscript.

\section{Karger'}




\section{Data Availability Statement}

The datasets used in the current study are available from the corresponding author on reasonable request.

\section{References}

1 Gilman S, Wenning GK, Low PA, Brooks DJ, Mathias CJ, Trojanowski JQ, et al. Second consensus statement on the diagnosis of multiple system atrophy. Neurology. 2008;71:670-6.

2 Wenning GK, Tison F, Ben Shlomo Y, Daniel SE, Quinn NP. Multiple system atrophy: a review of 203 pathologically proven cases. Mov Disord. 1997;12:133-47.

3 Ben-Schlomo Y, Wenning GK, Tison F, Quinn NP. Survical of patients pathologically proven multiple system atrophy. Neurology. 1997;48:384-93.

4 Watanabe H, Saito Y, Tarao S, Ando T, Kachi T, Mukai E, et al. Progression and prognosis in multiple system atrophy. An analysis of 230 Japanese patients. Brain. 2002;125:1070-83.

5 Wenning GK, Geser F, Krismer F, Seppi K, Duerr S, Boesch S, et al. The natural history of multiple system atrophy: a prospective European Cohort Study. Lancet Neurol. 2013;12:264-74.

6 Coon EA, Sletten DM, Suarez MD, Mandrekar JN, Ahlskog JE, Bower JH, et al. Clinical features and autonomic testing predict survival in multiple system atrophy. Brain. 2015;138:3623-31.

7 Halliday GM, Holton JL, Revesz T, Dickson DW. Neuropathology underlying clinical variability in patients with synucleinopathies. Acta Neuropathol. 2011;122:187-204.

8 Ozawa T, Paviour D, Quinn NP, Josephs KA, Sangha H, Kilford L, et al. The spectrum of pathological involvement of the striatonigral and olivopontocerebellar systems in multiple system atrophy: clinicopathologiucal correlations. Brain. 2004;127:2659-71.

9 Jellinger KA. More frequent Lewy bodies but less frequent Alzheimer-type lesions in multiple system atrophy as compared to age-matched control brains. Acta Neuropathol. 2007;114:299-303.

10 Braak H, Del Tredici K, Rüb U, de Vos RA, Jansen Steur EN, Braak E. Staging of brain pathology related to sporadic Parkinson's disease. Neurobiol Aging. 2003;24:197-211.

11 Miki Y, Foti SC, Asi YT, Tsushima E, Quinn N, Ling H, et al. Improving diagnostic accuracy of multiple system atrophy: a clinicopathological study. Brain. 2019;142:2813-27.

12 Mochizuki A, Komatsuzaki Y, Shoji S. Association of Lewy bodies and glial cytoplasmic inclusions in the brain of Parkinson's disease. Acta Neuropathol. 2002;104:534-7.

13 Shimohata T, Aizawa N, Nakayama H, Taniguchi H, Ohshima Y, Okumura H, et al. Mechanisms and prevention of sudden death in multiple system atrophy. Parkinsonism Relat Disord. 2016;30:1-6.

14 ShimoharaT, Ozawa T, Nakayama H, Tomita M, Shinoda H, Nishizawa M, et al. Frequency of nocturnal sudden death in patients with multiple system atrophy. J Neurol. 2008;225:1483-5. 